\title{
Entre parole d'expert et visée programmatique : les policy briefs, genre discursif propre aux think tanks américains
}

Expert discourse and agenda-driven recommendations in policy briefs, a genre specific to American think tanks

\section{Mathilde Gaillard}

\section{OpenEdition}

Journals

Édition électronique

URL : https://journals.openedition.org/asp/4647

DOI : 10.4000/asp.4647

ISSN : 2108-6354

\section{Éditeur}

Groupe d'étude et de recherche en anglais de spécialité

Édition imprimée

Date de publication : 15 mars 2015

Pagination : 55-80

ISSN : 1246-8185

Référence électronique

Mathilde Gaillard, «Entre parole d'expert et visée programmatique : les policy briefs, genre discursif propre aux think tanks américains », ASp [En ligne], 67 | 2015, mis en ligne le 09 mars 2016, consulté le 21 septembre 2021. URL : http://journals.openedition.org/asp/4647 ; DOI : https://doi.org/10.4000/ asp.4647

Ce document a été généré automatiquement le 21 septembre 2021.

Tous droits réservés 


\title{
Entre parole d'expert et visée programmatique : les policy briefs, genre discursif propre aux think tanks américains
}

\author{
Expert discourse and agenda-driven recommendations in policy briefs, a genre \\ specific to American think tanks
}

Mathilde Gaillard

\section{Introduction}

1 Si le mot «think tank» désignait à la fin du XIX siècle une façon plutôt comique et négative de désigner le cerveau (vide) d'un individu (Medvetz 2009), il prend aujourd'hui un sens tout différent. Les think tanks, ou «boîtes à penser » ou encore « cercles de réflexion » si l'on suit l'appellation française, constituent en effet l'un des lieux privilégiés d'élaboration de la pensée politique aujourd'hui. Institutions indépendantes à but non lucratif rassemblant des experts de politique publique, ils se caractérisent par des activités de recherche et de production d'analyses dans le but d'enrichir le débat politique. Ces analyses sont destinées d'abord aux hommes politiques et à leurs équipes et secondairement aux médias et au grand public. Cette proximité avec le milieu politique, particulièrement remarquable dans le cas des think tanks américains ${ }^{1}$, a mené de nombreux auteurs à affirmer que ces organisations s'inscrivaient également dans une démarche d'influence (Abelson 2006; Boucher et al. 2012 ; Stone 1996).

2 Dans cette étude, nous reprenons l'hypothèse de ces auteurs sur la position spécifique des think tanks dans la société américaine. En raison de la nature de leur activité, les think tanks se caractérisent par une position unique à la frontière du monde de la recherche universitaire et du monde politique. En effet, ils produisent de la recherche, 
tout en cherchant, par la diffusion même de ces analyses, à orienter les politiques publiques.

3 Nous postulons que cette situation d'intermédiaire entre monde de la recherche et milieu politique se traduit par des contraintes d'origine interne et externe, auxquelles sont soumis les membres des think tanks américains dans leur pratique professionnelle. Ces contraintes créent une situation d'équilibre ou encore une tension entre deux champs que sont l'expertise et l'action politique.

4 Dans une première analyse des différents textes publiés par cinq think tanks américains sur leurs sites internet (Gaillard 2014), nous avons montré que les think tanks étudiés se caractérisaient par la production de genres discursifs communs, dont font notamment partie les policy briefs, courts documents spécifiquement destinés aux hommes politiques et répondant à leurs besoins d'analyse et de prévision.

5 La présente étude vise dès lors à caractériser l'incidence de la position spécifique des think tanks dans la société américaine sur les policy briefs. Nous envisageons l'hypothèse suivante : les contraintes liées à la position spécifique des think tanks et la tension entre expertise et action politique qui en résulte, influencent la nature du genre des policy briefs et les procédés rhétoriques qui y sont à l'œuvre.

6 Notre étude se déroule en trois temps. Dans une première section, nous analysons la position d'interface des think tanks américains entre monde de la recherche et monde politique et, plus spécifiquement, entre expertise et action politique. Nous précisons les contraintes qui en résultent. Nous présentons ensuite le genre des policy briefs, ainsi que le corpus étudié et la méthode retenue pour l'analyse de leurs procédés rhétoriques. Enfin, nous consacrons la troisième partie à la discussion des résultats obtenus.

\section{La position d'interface des think tanks américains : entre expertise et action politique}

$7 \quad$ Nés au début du $\mathrm{XX}^{\mathrm{e}}$ siècle sous l'impulsion de grandes organisations caritatives qui $\mathrm{y}$ voyaient un moyen de créer un environnement intellectuel stimulant, notamment en matière de politique étrangère, les think tanks n'ont réellement pris leur essor qu'après la Seconde Guerre mondiale. Le gouvernement américain commença à voir les avantages que pouvait lui procurer un ensemble d'experts sur des domaines aussi divers que l'ingénierie ou les sciences sociales et en encouragea la création. C'est cependant l'émergence d'une parole intellectuelle contestataire ${ }^{2}$ dans les années 1960 et 1970 qui favorisa la naissance des institutions tournées vers la promotion d'idées politiques que nous connaissons aujourd'hui.

8 L'institutionnalisation des think tanks dans le paysage politique américain s'explique également par la spécificité du système politique du pays ${ }^{3}$. À chaque changement d'administration, une majorité du personnel administratif se voit remplacée, permettant aux experts de ces institutions d'accéder aux plus hautes fonctions administratives (Abelson 2006 : 128). Ce phénomène, souvent qualifié de revolving door, contribue à ériger les think tanks en réservoirs de personnel administratif, et les rend dès lors particulièrement proches du pouvoir décisionnel. À l'inverse de la France où l'expertise technique est intégrée aux corps de l'État, on observe aussi plus généralement aux États-Unis un vide dans la production des connaissances au service de la décision publique, l'université étant davantage tournée vers la recherche 
fondamentale. Ceci, conjugué à l'absence d'une ligne politique forte au sein des partis politiques américains, rend la classe politique plus disposée à écouter des avis extérieurs (Geuens 2009). Les think tanks, dont les chercheurs, d'ailleurs généralement issus du monde universitaire, s'attachent à produire de la recherche au service des décisions politiques et du débat public, ont donc su s'emparer de ce vide.

Les think tanks occupent dès lors une position d'intermédiaire entre monde de la recherche et milieu politique ou, plus précisément, une situation à l'interface de plusieurs champs, que sont l'expertise et l'action politique.

Parce qu'elle répond aux besoins d'hommes politiques souvent non spécialistes, la recherche engagée par les think tanks doit être pragmatique, prendre en compte leurs préoccupations et suivre, voire anticiper, l'actualité :

Think tanks, like other organizations, need to renew their agendas from time to time. [...] they may have to shift the direction of their research in order to ensure that their work remains relevant to their nation's evolving policy agenda. [...] perhaps most fundamentally, in order to raise funds, organizations must offer to work on subjects for which there is a demand for new information and analysisthey must follow the market. (Struyk $2002: 73$ )

11 Les membres des think tanks ne peuvent donc être définis comme des chercheurs au sens classique du terme, mais s'apparentent plutôt à des experts. Ainsi, D. MacRae et D. Whittington (1997: 7) remarquent :

[a group of experts] resembles a scientific community, except that it is guided by practical rather than purely theoretical criteria of excellence and is concerned not only with internal standards but also with performing functions for, and thus interacting with, laymen.

12 Il convient de définir ce que nous entendons par expertise et par expert. Le Dictionnaire des politiques publiques la définit comme une activité orientée vers la résolution des problèmes; une situation d'expertise est alors «la rencontre d'une conjoncture problématique et d'un savoir spécialisé » (Saint-Martin 2010). Suivant la définition proposée par Anthony Saber (2014), nous avançons que les experts peuvent être considérés comme "des acteurs qui mettent les connaissances poussées qu'ils ont acquises, par leur formation ou leur expérience, au service d'une enquête ou d'une évaluation ». Les membres des think tanks répondent à cette définition dans la mesure où ils sont détenteurs à la fois de "savoirs disciplinaires » et de savoir-faire (Saber 2014). En effet, la plupart d'entre eux sont titulaires d'un doctorat et ont souvent fait carrière dans la grande administration, ce qui leur permet d'acquérir une certaine maîtrise des complexités du système politique américain (Medvetz 2009). C'est l'ensemble de ces compétences qui leur confère l'autorité intellectuelle nécessaire au statut d'expert.

13 À l'inverse des experts traditionnels dont la légitimité à intervenir dans l'espace public émane des demandes d'évaluation d'un mandataire, les think tanks sont eux-mêmes à l'initiative de leurs analyses. La légitimité d'un think tank à intervenir dans la sphère politique tient dès lors à l'attention que voudront bien lui accorder les hommes politiques. Bien que fondée historiquement, celle-ci demeure cependant fragile, dans la mesure où les décideurs sont sollicités par une multiplicité d'acteurs politiques tels que les groupes de pression, les cabinets de conseil ou encore les autres think tanks. Au cœur de l'activité d'expertise des think tanks américains se trouve le désir d'orienter, dans une certaine mesure, les débats politiques. En diffusant leurs analyses, ces institutions 
s'inscrivent dans une démarche d'influence à plus ou moins long terme. Elles sont donc engagées dans l'action politique.

La situation à la croisée de l'expertise et de l'action politique des think tanks américains nous semble se traduire à l'échelle de ces organisations par un certain nombre de contraintes, plus ou moins formalisées, auxquelles sont soumis leurs membres. Suivant la distinction élaborée par Michel Petit (2010), nous isolons deux types de contraintes ou « régulations » régissant les activités et l'organisation des think tanks : l'une émanant d'instances externes au think tank, l'autre, d'instances internes à l'organisation. Ces contraintes ou ces codes sont d'ailleurs explicitement exprimés dans certains manuels rédigés par des responsables de think tanks à destination des membres de ces institutions ${ }^{4}$.

Une première contrainte externe majeure pesant sur les think tanks américains concerne leur statut fiscal. Bien que les think tanks cherchent à " capturer l'imagination politique de la nation» comme le laisse par exemple entendre Diane Stone (1996), ils sont cependant à distinguer des groupes de pression qui ne cachent pas leur volonté d'intervenir directement dans le processus politique. Ils sont en effet considérés comme des organisations à visée non lucrative et non partisane, ce qui leur permet, ainsi qu'à leurs donateurs, d'être exonérés d'impôts (Abelson 2006: 79). Ils ne doivent pas entrer dans la promotion ouverte d'idées partisanes, mais faire montre d'une certaine objectivité.

Ce premier élément s'ancre dans une exigence plus large liée au besoin de légitimité de ces institutions. Bien qu'ils doivent rendre leurs idées visibles face à la concurrence sur le marché des idées, une incitation trop ouverte à l'action politique pourrait par exemple nuire à la crédibilité des think tanks auprès du public et des décideurs.

Cette obligation se traduit par une autre contrainte pesant sur les membres des think tanks, émanant cette fois de leurs instances internes. Dans la mesure où ils s'adressent bien souvent à des non-spécialistes du sujet débattu (hommes politiques, journalistes ou membres du grand public), les experts des think tanks doivent rendre leur propos accessible. Un membre d'un think tank observe à ce propos : « to be a successful think tank person, you need to be able to write in a way that is understandable to non-specialists" (Medvetz 2012 : 154). Un expert travaillant pour un think tank doit ainsi posséder la rigueur et le sérieux d'un universitaire et la capacité de s'adapter à son public, de l'interpeller et de rendre ses propos attractifs et adaptés à l'ordre du jour. Un certain nombre de think tanks ont d'ailleurs été amenés à produire plusieurs types de publications afin de répondre aux besoins divers de leur public ${ }^{5}$. Cette pluralité des compétences est bien souvent exigée par les responsables des think tanks eux-mêmes, comme le constate un président de think tank: "[w] really want you to try and always have one foot in the analytic camp and one foot in the advocacy camp " (Medvetz 2012:163). Ceci conduit alors un expert de la Brookings Institution à confier : "[w]e're Janus-faced, looking in both directions » (Medvetz $2012: 41$ ).

18 L'ensemble de ces contraintes place donc les think tanks dans une situation de tension entre deux pôles, ceux de l'expertise et de l'action politique.

Nous postulons que la notion de tension est inhérente à l'activité d'expertise. Dans le cas où ils interviennent dans un espace autre que le leur, les experts sont nécessairement amenés à dépasser le monde dans lequel ils évoluent et de fait à en «transgresser les codes» (Robert 2008:316). Leur légitimité à intervenir tient cependant à leur appartenance au monde scientifique. Toute activité d'expertise 
semble ainsi marquée par une tension, une ambivalence entre le monde de l'expertise et le monde dans lequel il intervient. Il nous semble que cette tension est d'autant plus marquée dans les think tanks américains que ces derniers ne sont pas réellement sollicités pour intervenir dans le monde politique et doivent faire face à la concurrence des autres acteurs politiques. Nous émettons donc l'hypothèse que les contraintes soulignées naissent du besoin des experts des think tanks de préserver leur légitimité dans le milieu politique américain. Il leur est en effet nécessaire de développer un certain nombre de stratégies pour rendre visibles leurs idées, sans pour autant s'inscrire dans une démarche politique clairement partisane qui nuirait à leur crédibilité.

Ces exigences liées à la position des think tanks à la frontière de l'expertise et de l'action politique nous paraissent également se répercuter sur certains phénomènes rhétoriques observés dans leur discours. Nous explorons cette hypothèse en nous appuyant sur un corpus de policy briefs publiés par trois think tanks américains.

\section{Constitution du corpus et méthode}

\subsection{Choix des think tanks}

21 Nous avons constitué un corpus rassemblant des policy briefs produits par trois des plus importants think tanks américains, et de tendances politiques différentes: la Brookings Institution, la Heritage Foundation et le Center for American Progress.

Créée en 1927, la Brookings Institution constitue l'un des think tanks américains les plus anciens. Souvent qualifiée de "university without students ", elle est sans doute, parmi les organisations à l'étude, la plus tournée vers la recherche. La Heritage Foundation symbolise, quant à elle, l'émergence des think tanks conservateurs dans les années 1970, supports idéologiques d'un mouvement à l'époque en plein essor. Créé en 2003 par John Podesta, ancien conseiller du président Clinton, le Center for American Progress représente une forme de "riposte libérale » visant à contrecarrer la forte proportion de think tanks conservateurs dans le paysage politique américain (Boucher et al. 2012 : 57-58).

Ces trois organisations figurent parmi les think tanks les plus fréquemment cités par les auteurs de monographies sur le sujet (Abelson 2006; Boucher et al. 2012; Medvetz 2012). Ils ont été sélectionnés, dans le cadre de notre étude, en fonction de leur taille et de leur réputation qui transparaissent notamment au travers de l'importance de leur budget et de leurs équipes. Nous nous sommes appuyée sur les données contenues dans le NIRA's World Directory of Think Tanks publié en 2005 qui établit une liste des think tanks du monde entier et de leur profil (2005), ainsi que sur les rapports annuels publiés par chacune des institutions. Le rapport du NIRA nous a permis d'obtenir la répartition exacte des membres du think tank, car les rapports annuels demeurent vagues à ce sujet. L'orientation politique du think tank, mentionnée sur les sites des institutions, a été également un critère de sélection afin d'obtenir un panel de tendances politiques variées. Ces données sont résumées dans le tableau 1. 
Tableau 1. Budgets annuels et profils des trois think tanks américains choisis

\begin{tabular}{|l|l|l|l|}
\hline & $\begin{array}{l}\text { Budget (en million de } \\
\text { dollars) }\end{array}$ & $\begin{array}{l}\text { Équipe } \\
\mathbf{( 2 0 0 5}^{6}\end{array}$ & $\begin{array}{l}\text { Tendance } \\
\text { politique }\end{array}$ \\
\hline Brookings Institution (BI) & $95,3(2013)$ & $\begin{array}{l}117 \mathrm{RF}, 160 \mathrm{E}, \\
60 \mathrm{NRF}^{7}\end{array}$ & Centriste \\
\hline Heritage Foundation (HF) & $84,9(2013)$ & $61 \mathrm{RF}, 134 \mathrm{E}$ & Conservatrice \\
\hline $\begin{array}{l}\text { Center for American Progress } \\
\text { (CAP) }\end{array}$ & $10(2005)$ & $89 \mathrm{RF}$ & Libérale ${ }^{8}$ \\
\hline
\end{tabular}
désigner les textes de notre corpus : les initiales du think tank suivies du sujet abordé dans le document (par exemple: "Youth Unemployment») et de sa date de publication.

\subsection{Les policy briefs : un genre}

Les policy briefs constituent l'un des genres communément diffusés par les think tanks américains sur leurs sites. Ce genre englobe en réalité plusieurs textes aux dénominations différentes selon les think tanks: les backgrounders (Heritage Foundation), les issue briefs (Center for American Progress), ou encore les papers (American Enterprise Institute, Cato Institute). Tous ces textes partagent cependant une même visée communicationnelle. Leur nature, leurs objectifs, leurs moyens de diffusion et leurs types de public sont similaires. Ils consistent en des rapports d'une dizaine de pages, diffusés sur les sites institutionnels des différents think tanks et qui s'adressent aux hommes politiques et à leurs équipes en réponse à un besoin d'information, d'analyse et de prévision. Les policy briefs sont particulièrement adaptés aux besoins des hommes politiques car ils répondent aux contraintes de temps auxquelles ils sont soumis (Abelson 2006 : 85). En effet, selon la Heritage Foundation, qui fut la première à produire ce genre de rapports, leur longueur est notamment déterminée par le «test de la valise » :

As one Heritage fellow puts it, "The test was if the Congressman could put it in his briefcase and read it going to the National Airport...then okay, he might find some use in it and take the arguments and rely upon those in the debate about a particular issue". (Medvetz $2012: 120$ )

Pour les désigner, nous avons donc choisi d'employer le nom de policy brief, dénomination la plus fréquente trouvée dans les travaux sur les think tanks américains.

C'est précisément cette visée communicationnelle commune qui nous a permis d'identifier ces textes comme constituant un même genre discursif et d'en faire notre objet d'étude (Gaillard 2014). En effet, la notion de genre peut être envisagée du point de vue des « buts poursuivis» (Resche 2005). Ainsi, John Swales observe :

The principal criterial feature that turns a collection of communicative events into a genre is some shared set of communicative purposes. $(1990: 46)$

Nous avons ainsi constitué un corpus de soixante-huit policy briefs rédigés par les trois think tanks cités, soit environ une vingtaine de textes par organisation (voir liste 
complète en annexe 2). Les documents portent sur des sujets divers et ont été publiés entre janvier 2010 et novembre $2013^{9}$.

\subsection{Méthode d'exploitation du corpus}

29 Notre étude s'inscrit dans le cadre général d'une analyse de genre. Nous adoptons une approche modulaire qui combine plusieurs niveaux ou «modules» d'analyse, nous permettant de conserver une vision générale du genre étudié, ou encore selon Henning Nölke :

[...] une approche qui a recours à un modèle théorique contenant un certain nombre de sous-systèmes autonomes appelés modules, où chaque module est chargé du traitement d'une problématique restreinte. (2006: 175)

Plusieurs modules peuvent être envisagés dans le cadre d'une analyse de genre: module structurel, interactionnel, discursif, stylistique ou encore lexical. Pour les besoins de cette étude, nous avons cependant retenu deux niveaux transversaux d'analyse au sein de ces modules, à savoir la macro-structure du policy brief ainsi que les phénomènes rhétoriques qui peuvent y être observés.

31 L'étude de la macro-structure des policy briefs vise à identifier le mouvement argumentatif général du genre. En effet, Vijay Bhatia note: «each move serves a typical communicative intention which is always subservient to the overall communicative purpose of the genre » (1993 : 37). L'identification de ces mouvements a été conduite manuellement pour chacun des policy briefs étudiés.

32 L'analyse de certains aspects rhétoriques des policy briefs s'attache à préciser leurs stratégies argumentatives. Nous émettons en effet l'hypothèse que les contraintes auxquelles sont soumis les think tanks américains influencent la macro-structure et les procédés rhétoriques à l'œuvre dans l'un des genres qu'ils produisent. Il s'agit d'établir si le genre des policy briefs peut être considéré comme le lieu d'une tension entre deux dimensions, l'une relevant de l'expertise et l'autre, plus programmatique, visant à influencer les acteurs du monde politique.

33 Nous proposons une étude des phénomènes interactionnels qui s'y déploient, souvent désignés sous le concept d'évaluation ou «evaluation" (Hunston \& Thompson 2000 ; Hyland 2005). Il fait référence aux jugements, sentiments ou positionnements de l'auteur dans un texte. Ces jugements et opinions ne prennent cependant sens que dans la mesure où ils font référence au système de valeur d'une communauté, d'où la dimension interactionnelle du concept d'évaluation (Hyland 2005). Ainsi, Susan Hunston et Geoff Thompson (2000) remarquent: "[e]very act of evaluation expresses a communal value system and every act of evaluation goes towards building up that value-system ». Un auteur ne peut dès lors être convaincant que lorsqu'il se positionne vis-à-vis de ce système de valeur. L'étude de l'évaluation dans un texte permet ainsi de définir les valeurs de son auteur et de la communauté à laquelle il appartient et, par conséquent, d'identifier les ressorts de son argumentaire. Elle nous paraît, à ce titre, pertinente pour mettre en lumière les procédés rhétoriques qui se déploient dans les policy briefs. Le concept d'évaluation peut recouvrir divers phénomènes textuels, tels que la manifestation de la présence de l'auteur, de son positionnement, qu'il soit affectif ou épistémique, ou encore de sa relation avec le lecteur. Ils sont alors réalisés de diverses façons sur le plan linguistique. Nous avons choisi ici de nous concentrer exclusivement 
sur les marqueurs d'attitude, d'une part et les pronoms personnels de première personne référant aux auteurs des policy briefs, d'autre part.

Les marqueurs d'attitude traduisent le positionnement affectif ou encore le jugement de l'auteur dans son texte. Les policy briefs consistant en des analyses argumentées de politiques données, ils sont particulièrement pertinents pour l'étude des phénomènes rhétoriques qui s'y déploient. En effet, Ken Hyland remarque :

By signalling an assumption of shared attitudes, values and reactions to material, writers both express a position and pull readers into a conspiracy of agreement so that it can often be difficult to dispute these judgements. $(2005: 180)$

Nous nous proposons d'effectuer un relevé manuel de ces marqueurs d'attitude dans le corpus de policy briefs à l'étude. Ces marqueurs se réalisent sur un ensemble assez large de catégories grammaticales, ainsi que sur plusieurs niveaux, tels que ceux du lexique, de la phrase ou des propositions subordonnées (Biber et al. 1999). Ils peuvent prendre la forme d'adjectifs comme dans l'exemple (1), d'adverbes, de verbes ou encore de tournures plus longues comme on l'observe l'exemple (2):

1) This notion that the system worked until banks foreclosed on millions of homes is inaccurate ${ }^{10}$. (HF Finance novembre 2010)

2) The Obama administration and Congress deserve credit for showing the foresight to allocate resources to these programs, which are rooted in a rich body of evidence demonstrating their efficacy and their cost-effectiveness. (BI Pregnancy mars 2012)

Il n'est néanmoins pas aisé d'identifier ce qui relève d'une prise de position de l'auteur et ce qui n'en relève pas. C'est notamment le cas des marqueurs d'attitude présents dans du discours rapporté au mode indirect :

3) [I]n the most recent Gallup survey, a record 76 percent of respondents said that most members of Congress do not deserve re-election. (BI Political Reform 2012)

Dans la mesure où nous ne nous intéressons qu'au point de vue de l'auteur dans notre corpus, ces marqueurs, parce qu'ils relèvent du point de vue des personnes interrogées, pourraient ne pas être pris en compte dans notre relevé. Cependant, l'auteur du policy brief a fait le choix de mentionner cet élément particulier d'information et, de fait, a pris position vis-à-vis d'un tel constat (Charles 2006 : 494). Les marqueurs présents dans ce type de discours rapporté ont donc été pris en compte lors de notre relevé. Le contexte d'apparition des marqueurs d'attitude est ainsi crucial lors de leur identification. Il convient cependant de noter que nous ne pouvons prétendre à l'exhaustivité. En effet, l'attitude peut se traduire dans un texte sous des formes aisément identifiables telles que des mots ou des tournures, mais elle se révèle également sous divers aspects difficilement repérables pour une personne extérieure à la communauté professionnelle de l'auteur. L'absence ou la présence d'un élément considéré comme clé par la communauté scientifique de l'auteur constitue par exemple pour K. Hyland un marqueur d'évaluation à part entière (2005: 176).

Une lecture qualitative des policy briefs ainsi qu'une étude plus ciblée du sémantisme des marqueurs relevés, de leurs éventuels synonymes et antonymes, nous permettent d'identifier différentes thématiques ou encore divers réseaux notionnels dans les analyses proposées par leurs auteurs. Ce sont ces réseaux que nous nous proposons 
d'analyser dans cette étude. Par exemple, l'adjectif «effective » a été identifié comme relevant du thème de l'efficacité; ses synonymes tels que "efficient» ou "productive " ainsi que ses antonymes tels qu' "ineffective » ou "inefficient » appartiennent au même réseau notionnel ${ }^{11}$. Dans certains cas, le contexte textuel d'apparition de ces marqueurs se révèle déterminant pour en identifier la valeur sémantique et les intégrer ou non à un réseau notionnel, comme par exemple celui du «bon sens »:

4) Both the President and the Republican nominee have an obligation to engage in a realistic, fact-based debate on the budget options facing the nation and this requires acknowledgement of the reality the next president will face. (BI Economic Growth 2012)

41 L'emploi de l'adjectif « realistic » réfère ici à la notion de bon sens, qualité dont doivent faire preuve, selon l'auteur, les deux acteurs politiques mentionnés face à l'ampleur de la situation budgétaire des États-Unis. Par «bon sens », nous entendons la capacité de prendre une décision, sans a priori et de façon raisonnable.

L'identification de ces réseaux de sens nous permet dès lors de déterminer les problématiques à l'œuvre dans les jugements ou évaluations portés par les auteurs des policy briefs étudiés et ainsi de mettre en lumière certains procédés rhétoriques.

Une étude des pronoms personnels de première personne, témoins de la présence de l'auteur dans son texte, complète notre analyse des phénomènes interactionnels dans les policy briefs. Dans la mesure où la majorité des pronoms personnels de première personne de notre corpus relève de la première personne du pluriel ${ }^{12}$, nous nous sommes concentrée sur l'identification manuelle des différents référents du «we » et des pronoms associés «our » et «us» employés dans les policy briefs. Ils ont été classés en deux catégories : le «we » inclusif c'est-à-dire référant soit à l'auteur, soit au lecteur, soit à l'auteur et au lecteur, et le "we» exclusif référant soit à l'auteur et à des personnes extérieures, soit à une entité collective indéfinie (Quirk et al. 1985). Afin d'identifier les référents de ces pronoms le plus précisément possible, nous nous sommes appuyée sur le contexte dans lequel ces pronoms se trouvaient. En effet, K. Proctor et L.-W. Su remarquent :

[w]e cannot ascertain a personal pronoun's meaning within a single sentence; the meaning of a pronoun is established above the syntactical level. $(2011: 3253)$

Dans l'exemple suivant, c'est la valeur pragmatique de la phrase annonçant le plan du policy brief qui nous permet de conclure que le pronom « we » réfère exclusivement à ou aux auteur(s) du document et constitue de fait un « we» inclusif :

5) After reviewing the evidence, we suggest possible responses at the state and federal levels. (CAP Health novembre 2013)

De même, c'est le contexte textuel et notamment la comparaison, dans l'exemple (6), entre le peuple chinois et le peuple américain, qui nous permettent d'affirmer dans l'exemple suivant que le pronom, exclusif cette fois, réfère au peuple américain :

6) Then, like the Chinese, we can leverage a stronger, smarter grid to decrease our dependence on foreign oil, to drive innovation, to create jobs [...]. (CAP China Development octobre 2011)

Notre analyse des phénomènes interactionnels à l'œuvre dans les policy briefs est à la fois qualitative et quantitative. L'intégration d'une dimension quantitative dans notre 
étude nous permet, notamment dans le cas des marqueurs d'attitude, de mettre en lumière l'importance de certains réseaux notionnels et dès lors, la pertinence de certaines problématiques au sein des analyses proposées. Nous traitons cette dimension à l'aide du concordancier AntConc 3.2.4w 2011.

\section{Résultats}

\subsection{Macro-structure : une réponse à une demande d'expertise} étudiés. Cependant, l'organisation générale des documents concernés correspond à un agencement thématique dont chaque partie reprend la structure générale observée dans le reste du corpus. Dans un policy brief du Center for American Progress dédié à l'étude des propositions du mouvement conservateur le Tea Party (CAP Tea Party septembre 2011), nous retrouvons par exemple une introduction ainsi qu'une conclusion comportant quelques recommandations, mais les mouvements habituels (bilan et conséquences) sont organisés de façon thématique selon les propositions du Tea Party.

50 Nous pouvons conclure que les policy briefs obéissent à une structure extrêmement codifiée. Celle-ci suit d'ailleurs un schéma argumentatif plus large. Les policy briefs débutent en effet régulièrement par l'identification d'une situation problématique pour mieux en montrer les conséquences et proposer des solutions. C'est précisément l'identification de cette situation problématique qui légitime l'intervention des experts des think tanks dans l'espace public. La présence d'un problème est en effet le fondement de toute activité d'expertise. Ceci permet dès lors aux auteurs des policy briefs d'introduire, de façon logique et argumentée, leurs propositions visant à surmonter les difficultés identifiées et de fait, de convaincre leurs lecteurs de leur bienfondé.

51 La nature codifiée de la structure des policy briefs peut être également liée à la contrainte que représentent les attentes des hommes politiques, destinataires visés par ce type de rapport. Ceux-ci recherchent en effet des analyses rigoureuses et objectives leur permettant, à leur tour, de légitimer leurs décisions politiques comme fondées, 
justes et débarrassées de toute forme d'arbitraire. Cécile Robert note à ce propos (2008: $312)$ : «l'expertise naturalise la décision en la présentant comme la simple application de principes reconnus comme neutres et universels ». En utilisant une structure du type « analyse - conséquences - solutions », les auteurs des policy briefs répondent donc à cette exigence de rigueur et d'objectivité. Leurs analyses constituent dès lors des outils immédiatement exploitables.

\subsection{Procédés rhétoriques : témoins d'une visée programmatique}

52 À l'examen du sémantisme des marqueurs d'attitude observés dans le corpus ${ }^{14}$, nous avons pu identifier divers réseaux notionnels dans les policy briefs. Les résultats sont présentés dans les tableaux 2 et 3.

Tableau 2. Occurrence des différents réseaux notionnels identifiés au sein des marqueurs d'attitude relevés

\begin{tabular}{|l|l|l|}
\hline Réseaux notionnels & Nombre d'occurrences & $\begin{array}{l}\text { Fréquence } \\
\text { Marqueurs } \\
\text { d'attitude }\end{array}$ \\
\hline Urgence/nécessité & 448 & $17 \%$ \\
\hline Danger/anxiété & 400 & $16 \%$ \\
\hline Efficacité & 303 & $12 \%$ \\
\hline Réussite & 269 & $10 \%$ \\
\hline Pertinence & 218 & $9 \%$ \\
\hline Bon sens & 181 & $7 \%$ \\
\hline Difficulté & 129 & $5 \%$ \\
\hline Autres & 615 & $24 \%$ \\
\hline Total & 2563 & $\mathbf{1 0 0} \%$ \\
\hline
\end{tabular}


Tableau 3. Lexique porteur de marques d'attitude au sein de chaque réseau notionnel

\begin{tabular}{|c|c|c|c|c|c|c|}
\hline $\begin{array}{l}\text { Urgence/ } \\
\text { Nécessité }\end{array}$ & $\begin{array}{l}\text { Danger/ } \\
\text { Anxiété }\end{array}$ & Efficacité & Réussite & Pertinence & Bon sens & Difficulté ${ }^{15}$ \\
\hline Essential & Anxiety* & Costly & Success* & A bad idea & Clear & Complex \\
\hline It is time & Concern & Duplicative & To fail* & Acceptable & Credible & Complicated \\
\hline Now & Dangerous & Effective ${ }^{*}$ & & Accurate & Irresponsible & Difficult* \\
\hline Pressing & Disast* & Efficient & & Adequate & Meaningful & Hard" \\
\hline To need ${ }^{* 16}$ & Ominous & Ineffective & & Appropriate & Meaningless & Problem* \\
\hline Unnecessary & Risk* & Inefficient & & Consistent & Realistic & Simpl* \\
\hline Urgent & Safe & Innovative & & Correct & Responsible & Wors" \\
\hline \multirow[t]{14}{*}{ Vital } & Threat* & Productive & & Fair & Sense & \\
\hline & To harm* & Stal* & & Inaccurate & Sensible & \\
\hline & To $100 \mathrm{~m}^{*}$ & Sufficient & & Inadequate & Sound & \\
\hline & To undermine ${ }^{*}$ & Sustainable & & Inconsistent & Unrealistic & \\
\hline & Worry* & Unproductive & & Incorrect & Wise & \\
\hline & & Unsustainable & & Misguided & & \\
\hline & & Viable & & Misleading & & \\
\hline & & Waste* & & Mistake* & & \\
\hline & & & & Right & & \\
\hline & & & & To deserve* & & \\
\hline & & & & To jeopardize* & & \\
\hline & & & & To merit* & & \\
\hline & & & & Unacceptable & & \\
\hline & & & & Wrong & & \\
\hline
\end{tabular}

On note que $76 \%$ des marqueurs relevés ont pu être classés suivant sept réseaux notionnels principaux ${ }^{15}$; ils marquent ainsi l'importance de certaines problématiques dans les policy briefs. On observe également que certains réseaux notionnels, comme celui de la réussite, reposent sur une faible variété de mots; cependant le nombre d'occurrences de ces mots nous permet de l'inclure parmi les réseaux notionnels les plus fréquemment observés. Nous constatons que certains de ces réseaux notionnels sont proches sémantiquement, comme par exemple ceux de l'efficacité, de la réussite ou de la pertinence et qu'ils marquent ainsi la récurrence de certaines problématiques dans les évaluations proposées par les experts des think tanks étudiés.

Il convient donc de montrer en quoi ces réseaux notionnels nous permettent d'identifier ces problématiques et, plus largement, quels sont les procédés rhétoriques à l'œuvre dans les analyses proposées par les auteurs des policy briefs.

\subsubsection{Des politiques analysées comme problématiques et dangereuses : une forme d'incitation à l'action}

La proportion la plus importante des marqueurs relevés $(31 \%)$ correspond aux réseaux notionnels de l'efficacité, de la réussite et de la pertinence et témoigne des critères d'évaluation retenus par les experts des think tanks lors de leurs analyses:

7) In all, there are more than 150 federal education programs through which federal education spending is inefficiently and wastefully filtered back to the states. President Obama's budget does not eliminate ineffective and duplicative programs and further expands the federal government's bureaucratic and burdensome role in education. (HF Education avril 2012) 8) Nonetheless, the administration successfully adopted policies to protect public health from air pollution, lower oil consumption, and create jobs. (CAP Energy janvier 2013)

Les politiques évaluées dans nos exemples doivent, selon les experts, être efficaces et pertinentes en termes de coûts (7) et de résultats (8). Sont ainsi établis des bilans des politiques actuelles en fonction de leur réussite, de leur pertinence ou de leur efficacité. 
Ces bilans s'appuient notamment sur des données chiffrées et sur des recherches approfondies, comme nous l'avons montré lors d'une précédente étude (Gaillard 2014).

9) Unfortunately, Republicans in Congress are moving in the opposite direction, scaling back our nation's investment in community colleges and job training in FY 2011 and giving every indication that more of the same is on the agenda for FY 2012. That's a mistake. (CAP US Workforce avril 2011)

Dans la mesure où les membres des think tanks répondent à un besoin d'expertise, nous aurions pu nous attendre à ce que le discours relevé dans les policy briefs relève du ton neutre, objectif et factuel traditionnellement associé à l'activité d'expertise, ainsi que le note par exemple Andrew Rich : " experts have typically been thought of as neutral, credible and above the fray of the rough and tumble of policy making " (2004:2). Le ton des experts est ici pourtant extrêmement tranché et relève de jugements de valeur.

Les analyses proposées par ces experts sont par ailleurs systématiquement présentées comme critiques, notamment au vu des énumérations de l'exemple (7) ou de l'exemple (9). Si le succès des politiques analysées dans l'exemple (8) est reconnu, l'emploi de la conjonction de coordination à valeur adversative "nonetheless " souligne les limites d'un tel succès. Cette forte dimension critique nous paraît être inhérente aux policy briefs, comme le remarque Nadia Marzouki (2011:36) : « [u]n des traits récurrents du discours des think tanks est la critique de l'incohérence des politiques en cours, élaborées au coup par coup, de façon improvisée ". L'accent placé sur une situation problématique permet en effet aux auteurs d'interpeller le lecteur et de le mettre dans une position d'écoute des opinions avancées dans les policy briefs.

61 Un tel procédé rhétorique est d'autant plus frappant à l'examen des marqueurs d'attitude relevant de l'urgence et de l'anxiété, qui constituent les marqueurs les plus fréquemment relevés (33\%) :

10) However, a recent wave of activity at the state and federal levels most notably, Congress's attempt to defund Planned Parenthood and several states' efforts to do the same threaten to reverse much of this progress. (BI Pregnancy Mars 2012)

11) These cuts have been put forth with little consideration for their long-term impact: a dangerous degradation of America's capacity to deter, defend, and defeat her enemies. (HF Defence Cuts décembre 2011)

Dans les deux exemples, les auteurs présentent les mesures actuelles prises par l'exécutif comme néfastes, voire dangereuses, pour le bien du pays. On note ici à nouveau le registre dramatique employé par l'expert pour décrire la situation.

Les situations analysées sont alors présentées comme nécessitant une intervention de toute urgence : 
12) With the specter of fiscal austerity looming as federal, state, and local governments face budget deficits it is time to look for innovative and costeffective strategies to address our most pressing problems. (CAP Public Agencies février 2013) première personne du pluriel, apportent un éclairage supplémentaire à la visée programmatique des policy briefs. L'identification de leurs référents nous permet en effet d'analyser la façon dont l'auteur s'inscrit et interagit avec son lecteur dans son texte. Les résultats sont présentés dans le tableau 4.

Tableau 4. Répartition des fréquences des référents de la première personne du pluriel

\begin{tabular}{|l|l|}
\hline Référents We/our/us & $\begin{array}{l}\text { Fréquence Nombre total de } \\
\text { We/our/us \% }\end{array}$ \\
\hline Peuple américain & 65.68 \\
\hline Autre(s) ${ }^{16}$ & 17.26 \\
\hline Auteur(s) & 11.79 \\
\hline Ambigu & 4.00 \\
\hline Lecteur \& Auteur & 1.26 \\
\hline
\end{tabular}

67 Nous avons pu identifier trois types de référents pour le pronom « we » et ses corrélats « our » et « us » dans les policy briefs étudiés : le ou les auteur(s), l'auteur et le lecteur et le peuple américain. Nous observons qu'une forte majorité des marqueurs de première 
personne du pluriel fait référence au peuple américain, comme l'illustre la comparaison entre États-Unis et Grèce dans l'exemple suivant.

13) With around 10,000 Baby Boomers retiring every day for the next three decades, and nearly all of them qualifying for Social Security and Medicare, unless the federal government undergoes a profound shift in its spending and tax policy, we will soon be Greece. (BI Budget 2012)

Ce type de pronoms apparaît régulièrement en co-occurrence avec des auxiliaires de modalité, dans des contextes proposant des solutions ou alternatives aux politiques analysées :

14) We should continue to push for a diverse portfolio of cleaner energy while also exploring the safest ways to tap these new reserves using hydraulic fracturing. (CAP Fracking mars 2010)

15) We need to reimagine a world where, within 15 to 20 years, most people (at least 5 billion) will have such a lifestyle. (BI Private Sector septembre 2013)

Les auxiliaires de modalité tels que «need" ou "should» après le pronom "we » réfèrent au devoir ou à la nécessité d'agir du peuple américain. Celui-ci est incité à s'unir et à initier le changement proposé par l'expert. Bien que nous puissions envisager que le pronom de première personne du pluriel réfère uniquement aux membres des instances décisionnelles américaines, seules capables de prendre de telles décisions, il est difficile de distinguer ces deux entités. Un tel usage du " we » témoigne en effet d'un procédé rhétorique plus large visant à inclure le lecteur dans l'argumentaire mené par l'expert.

70 Les lecteurs des policy briefs sont par définition intéressés par l'état de leur pays, c'est pourquoi ils cherchent des analyses fouillées et des solutions dans les documents publiés par les think tanks. Par l'emploi du «we» exclusif, lecteur et auteur sont alors unis par une volonté commune, celle d'améliorer l'état de leur pays. Ainsi, K. Hyland note à propos de la première personne du pluriel $(2005: 182):$ « [i]t sends a clear signal of membership by textually constructing both the writer and the reader as participants with similar understanding and goals ».

71 Un tel jeu sur un sentiment de communauté fait écho aux stratégies rhétoriques caractéristiques du discours politique décrites par Pierre Charaudeau: "l'homme politique est la voix de tous à travers sa voix et en même temps, il s'adresse à tous comme s'il n'était que le porte-parole de la voix d'un tiers » (2005: 75). Comme le font les hommes politiques, les experts des think tanks adoptent un "éthos de solidarité " (Charaudeau 2005), insistant sur un sentiment de communauté à l'heure où le pays est présenté comme étant en plein bouleversement. Ils jouent sur l'inquiétude de leurs compatriotes en décrivant la situation actuelle comme critique et nécessitant des solutions de toute urgence. Ceci leur permet dès lors de mieux convaincre leurs lecteurs du bien-fondé de leurs propositions et de légitimer leur intervention.

Les solutions offertes dans les policy briefs sont dès lors présentées par les experts comme s'inscrivant dans l'intérêt du pays et relevant de la nécessité et du bon sens. Nous observons en effet que les analyses des think tanks étudiés se caractérisent notamment par ces réseaux notionnels : 
16) The Employment Non-Discrimination Act, or ENDA, is sorely needed. Gay and transgender workers face extraordinarily high rates of discrimination on the job, inflicting significant economic pain on many workers at a time when all families are struggling to stay afloat. (CAP Discrimination juin 2012) 17) Additionally, it would be wise to move slowly on standards because technology changes quickly and statically written standards could stifle innovation. (HF Cyber Security juin 2012)

Bien qu'éminemment politiques, les propositions suggérées par les auteurs sont présentées comme étant nécessaires et sages, par exemple pour qu'une certaine justice sociale soit préservée (16), objectif qui semble parfaitement acceptable pour tout lecteur. Nécessaires pour le bien du pays, ces solutions sont présentées comme allant de soi et, de fait, difficilement contestables. Leur intervention à la suite d'une série d'analyses chiffrées et d'un argumentaire rigoureux les fait paraitre comme légitimes et renforce la crédibilité de leur auteur. Le lecteur se voit ainsi indirectement incité à agir suivant ces propositions.

\section{Conclusion}

L'étude du genre des policy briefs des think tanks américains nous a permis de caractériser l'incidence de leur position spécifique dans la société américaine sur l'un des genres qu'ils produisent. Nous avons montré que les contraintes liées à la position d'interface des think tanks influencent les procédés rhétoriques caractéristiques du genre. L'étude de la macro-structure des policy briefs souligne leur visée argumentative générale, organisée pour introduire des solutions, mais également leur nature codifiée. Suivant un argumentaire rigoureux et étayé, les policy briefs répondent en effet aux besoins d'expertise de leurs destinataires. Cependant, l'étude des phénomènes interactionnels met en lumière l'insertion des experts des think tanks dans la sphère de l'action politique. La rhétorique de la crise et de l'urgence, mise au jour à travers l'étude des marqueurs d'attitude, permet à ces experts d'interpeller leurs lecteurs, de les inciter à l'action et, plus largement, de justifier leur intervention dans le monde politique américain. Leur statut fiscal, ainsi que leur besoin de crédibilité agissent cependant comme des contraintes sur la visée programmatique des policy briefs qui reste toujours indirecte. Les solutions proposées semblent ainsi résulter d'un argumentaire rigoureux et s'inscrire dans l'intérêt du pays, davantage que dans une vision politique spécifique. Le discours de l'expert constitue le soubassement idéal d'un tel argumentaire, fondé sur les notions de bon sens et de nécessité.

Nous pouvons donc conclure que les policy briefs sont le lieu d'une tension entre expertise et visée programmatique, elle-même témoin de la situation d'interface des think tanks dans la société américaine. À l'origine de cette tension se trouve, il nous semble, un fort désir des think tanks de conserver leur légitimité : s'il leur est nécessaire, pour exister sur le marché des idées, de rendre visibles leurs propositions auprès des hommes politiques américains, il leur faut, pour demeurer crédibles, atténuer la dimension programmatique de leurs écrits. Ce besoin de légitimité est d'autant plus marqué que les think tanks ne sont pas des experts traditionnels, mandatés pour délivrer leurs analyses. L'appui sur le statut de l'expert semble donc faire partie d'une stratégie de légitimation pour justifier l'intervention des think tanks dans le monde politique américain. Les textes qui résultent de cette activité d'expertise 
se caractérisent dès lors par leur nature hybride, à la croisée d'un discours scientifique et d'une visée programmatique.

Le cas des think tanks américains, à travers une étude plus large de leurs productions, nous permettrait d'étoffer la réflexion sur la nature de l'expertise, sa définition même, à l'heure où les experts jouent un rôle de plus en plus important dans l'espace public.

\section{BIBLIOGRAPHIE}

ABELSON, Donald. E. 2006. A Capitol Idea: Think Tanks and US Foreign Policy. Montreal; Ithaca [New York] : McGill-Queens University Press.

BHATIA, Vijay K. 1993. Analysing Genre : Language use in professional settings. Londres : Longman. BIBER, Douglas, Stig JOHANSSON, Geoffrey LEECH, Susan CONRAD \& Edward FINEGAN. 1999. Longman Grammar of Spoken and Written English. Harlow : Pearson Education Ltd.

BOUCHER, Stephen, Martine ROYO \& Pascal LAMY. 2012. Les think tanks, cerveaux dans la guerre des idées. Paris : Éditions du Félin.

Center for American Progress. 2014. « American Progress Experts », consulté le 16 avril 2014 <http://www.americanprogress.org/about/experts-alphabetical/>.

CHARAUDEAU, Pierre. 2005. Le discours politique : les masques du pouvoir. Paris : Vuibert.

CHARLES, Maggie. 2006. «The construction of stance in reporting clauses: a cross-disciplinary study of theses ». Applied Linguistics 27/3, 492-518.

Competitive Enterprise Institute. 2004. Field Guide for Effective Communication, consulté le 12 mars 2014 <http://cei.org/studies-books/field-guide-effective-communication>.

GEUENS, Geoffrey. 2009. « Avant-propos : think tanks, experts et pouvoir ». Quaderni. Communication, technologies, pouvoir 70, 5-9.

HUNSTON, Susan \& Geoff THOMPSON. 2000. « Evaluation: An Introduction ». In Hunston, S. \& G. Thompson (dir.), Evaluation in Text: Authorial Stance and the Construction of Discourse. Oxford : Oxford University Press, 5-27.

HYLAND, Ken. 2005. «Stance and engagement: a model of interaction in academic discourse ». Discourse Studies 7/2, 173-192.

GAILLARD, Mathilde. 2014. « Éléments de caractérisation du discours des think tanks américains, Approche par une étude de genre(s) ». Mémoire de Master 2 Recherche en Études anglophones, sous la direction d'Agnès Celle et d'Anthony Saber, soutenu à l'Université Paris 7-Diderot le 27 juin 2014.

MACRAE, Duncan Jr. \& Dale WhitTington. 1997. Expert Advice for Policy Choice: Analysis and Discourse. Washington D.C. : Georgetown University Press.

MARZOUKI, Nadia. 2011. « De l'endiguement à l'engagement : le discours des think tanks américains sur l'islam ». Archives de sciences sociales des religions 155/3, 21-39. 
MCGANN, James. 2012. « Global Trends in Think Tanks and Policy Advice ». Think Tanks and Civil Societies Program, International Relations Program, University of Pennsylvania.

Philadelphia : University of Pennsylvania Press.

MEDVETZ, Thomas. 2009. « Les think tanks aux États Unis : l'émergence d'un espace de production des savoirs ». Actes de la recherche en sciences sociales 176-177/1, 82-93.

MEDVETZ, Thomas. 2012. Think tanks in America. Chicago : University of Chicago Press.

NIRA's Center for Policy Research. 2005. NIRA's World Directory of Think Tanks 2005, consulté le 16 avril 2005 <http://www.nira.or.jp/past/ice/nwdtt/2005/>.

NÖLKE, Henning. 2006. « Linguistique modulaire et la linéarité de la langue ». Syntaxe et énonciation - Actes du XII Séminaire de Didactique universitaire, Constanta 2005, 173-204.

PAlTRIDGE, Brian. 2012. « Genre and ESP ». In Paltridge B. \& S. Starfield (dir.), The Handbook of

English for Specific Purposes. Malden, Ma : Wiley-Blackwell, 347-358.

PETIT, Michel. 2010 « Le discours spécialisé et le spécialisé du discours : repères pour l'analyse du discours en anglais de spécialité » E-rea, 8, consulté le 20 octobre 2013 <http://erea.revues.org/ $1400>$.

PROCTOR, Katarzyna \& Lily-Wen Su. 2011. « The first person plural in political discourse : American politicians in interviews and in a debate ». Journal of Pragmatics 43/13, 3251-3266.

RESCHE, Catherine. 2005. «Les mission statements des grandes entreprises cotées en Bourse : prélude à l'étude d'un genre particulier de textes dictés par plusieurs contextes ». ASp 47-48, consulté le 3 novembre 2014 <http://asp.revues.org/656>.

RICH, Andrew. 2004. Think Tanks, Public Policy, and the Politics of Expertise. New York: Cambrige University Press.

ROBERT, Cécile. 2008. «Expertise et action publique ». In Borraz, O. \& V. Guiraudon (dir.), Politiques Publiques 1. La France dans la gouvernance européenne. Paris : Presses de Sciences Po, 309-335.

SABER, Anthony. 2014. « Avant-propos ». ASp 65, consulté le 24 mars 2014 <http://asp.revues.org/ 4179>.

SAINT-MARTIN, Denis. 2010. « Expertise ». In Boussaguer L., S. Jacquot \& P. Ravinet (dir.), Dictionnaire des politiques publiques. Paris : Presses de Sciences Po, 266-273.

STONE, Diane. 1996. Capturing the Political Imagination, Think Tanks and the Policy Process. London : Routledge.

STRUYK, Raymond J. 2002. Managing Think Tanks: Practical Guidance for Maturing Organizations. Washington : The Urban Institute.

SWALES, John. M. 1990. Genre Analysis. Cambridge : Cambridge University Press.

QUIRK, Randolph, Sidney GREENBAUM, Geoffrey LEECH \& Jan SVARTVIK. 1985. A Comprehensive Grammar of the English Language. Harlow : Longman.

\section{ANNEXES}

Annexe 1. Classement des marqueurs d'attitude relevés par nature 


\begin{tabular}{|c|c|c|c|c|}
\hline \multicolumn{3}{|c|}{ Adverbes, adjectifs et participes passés } & \multirow{2}{*}{\begin{tabular}{|l|} 
Noms \\
at risk
\end{tabular}} & \multirow{2}{*}{$\begin{array}{l}\text { Verbes } \\
\text { to strengthen* }\end{array}$} \\
\hline adequate & ineffective & sophisticated & & \\
\hline aggressive* & inefficient & sound & concern & to do little \\
\hline ambitious & key & special & failure & to fail* \\
\hline appropriate* & large & specifically & lack & to harm* \\
\hline bad & limited & stable & problem & to threaten* \\
\hline basic & meaningful & striking & threat & to undermine* \\
\hline beneficial & merely & strong* & & To jeopardize* \\
\hline best & misguided & succesful* & & \\
\hline better & modest & sufficient & & \\
\hline careful* & necessary & surprisingly & & \\
\hline clear* & negative & sustainable & & \\
\hline compelling & only & too & & \\
\hline complex & perfect & tough & & \\
\hline comprehensive & poor & truly & & \\
\hline considerable & positive & typically & & \\
\hline consistent* & potential & unable & & \\
\hline costly & powerful & unacceptable & & \\
\hline credible & profitable & unclear & & \\
\hline dangerous & promising & unfortunately & & \\
\hline difficult & prudent & unique & & \\
\hline dramatic* & real & unnecessary & & \\
\hline duplicative & reasonable & unprecedented & & \\
\hline easier & regrettably & unsurprisingly & & \\
\hline easy* & relatively & unusually & & \\
\hline effective* & relevant & useful & & \\
\hline efficient & reliable & valuable & & \\
\hline
\end{tabular}




\begin{tabular}{|l|l|l|l|l|}
\hline even & reluctant & viable & & \\
\hline exaggerated & responsible & vigorous & & \\
\hline extreme & right* & weak & & \\
\hline fortunately & risky & well & & \\
\hline full & robust & wise & & \\
\hline good & sensible & worse & & \\
\hline great* & serious* & worst & & \\
\hline growing & severe* & worth* & & \\
\hline hard* & sharply & & & \\
\hline heavily & simple* & & & \\
\hline inadequate & skeptical & & & \\
\hline
\end{tabular}

\section{Annexe 2. Corpus d'étude}

\subsection{Brookings Institution}

ANDERSON, A. 2010. «Combating Climate Change Through Quality Education » [BI Climate Change septembre 2010]. Washington DC : Brookings Institution.

BAILY, M. 2011. « Adjusting to China: A Challenge to the US Manufacturing Sector » [BI China janvier 2011]. Washington DC : Brookings Institution.

BAILY, M. 2012. « Restoring Economic Growth » [BI Economic Growth 2012]. Washington DC : Brookings Institution.

BUSH, R. 2010. « China-Japan Security Relations » [BI China Japan]. Washington DC : Brookings Institution.

BUSH, R. 2013. « Uncharted Strait » [BI Taiwan janvier 2013]. Washington DC : Brookings Institution.

CARDENAS, M. \& J. MELTZER. 2011. « Korea, Columbia, Panama: Pending Trade Accords Offer Economic Strategic Gains for the United States » [BI Trade juillet 2011]. Washington DC : Brookings Institution.

COOK, P. \& J. LUDWIG. 2011. « More Prisoners Versus Crime is the Wrong Question » [BI Prisons décembre 2011]. Washington DC : Brookings Institution.

DYNAN, K., T. GAYER \& N. PLOTKIN. 2013. « The Recent Homebuyer Tax Credit: Evaluation and Lessons for the Future » [BI Tax Credit juin 2013]. Washington DC : Brookings Institution.

FERRIS, E. 2011. « Protecting Civilians in Disasters and Conflicts » [BI Disasters mars 2011]. Washington DC : Brookings Institution. 
GALSTON, W. 2012. « Reforming Institutions » [BI Political Reform 2012]. Washington DC : Brookings Institution.

GAYER, T. \& E. PARKER. 2013. « The Car Allowance Rebate » [BI Cash for Clunkers]. Washington DC : Brookings Institution.

HASKINS, R. 2012. « Addressing the Budget Deficit » [BI Budget 2012]. Washington DC : Brookings Institution.

JONES, B., T. WRIGHT \& J. ESBERG. 2012. « Reviving American Leadership » [BI US Leadership 2012]. Washington DC : Brookings Institution.

KHARAS, H. 2013. « Reimagining the Role of the Private Sector in Development » [BI Private Sector septembre 2013]. Washington DC : Brookings Institution.

MALONEY, S. 2012. « Keeping Iran in Check » [BI Iran 2012]. Washington DC : Brookings Institution.

O'HANLON, M. 2011. « Improving Afghan War Strategy » [BI Afghanistan février 2011]. Washington DC : Brookings Institution.

SAWHILL, E. 2012. « Are We Headed Toward a Permanently Divided Society? » [BI Society mars 2012]. Washington DC : Brookings Institution.

THOMAS, A. 2012. « Policy Solutions for Preventing Unplanned Pregnancy » [BI Pregnancy mars 2012]. Washington DC : Brookings Institution.

VAN DER GAAG, J. \& A. ADAMS. 2010. « Where is the Learning? Measuring Schooling Efforts in Developing Countries » [BI Education avril 2010]. Washington DC : Brookings Institution.

VILlASENOR, J. 2011. «The Comprehensive Patent Reform of 2011: Navigating the LeahySmith America Invents Act » [BI Patent Reform septembre 2011]. Washington DC : Brookings Institution.

WEST, D. 2011. « Creating a Brain Gain for US Employers: the Role of Immigration » [BI Immigration janvier 2011]. Washington DC : Brookings Institution.

WITTES, B. \& D. BYMAN. 2012. « Keeping on Offense » [BI Terrorism 2012]. Washington DC : Brookings Institution.

\subsection{Center for American Progress}

ALI, W. \& M. DUSs. 2011. « Understanding Sharia Law: Conservatives' Skewed Interpretation Needs Debunking » [CAP Sharia Mars 2011]. Washington DC : Center for American Progress.

AYRES, S. 2010. « The High Cost of Youth Unemployment » [CAP Youth Unemployment avril 2010]. Washington DC : Center for American Progress.

BURNS, C. \& J. KREHELY. 2012. « The Freedom to Work, The Freedom to Worship: The Employment Non-Discrimination Act Advances Workplace Equality and Protects Religious Liberty » [CAP Discrimination juin 2012]. Washington DC : Center for American Progress. 
CALEYNAND, M. \& E. OSHIMA LEE. 2013. « The Threat of Self-Insured Plans among Small Businesses » [CAP Small Businesses juin 2013]. Washington DC : Center for American Progress.

COCKMAN, C., B. KATULIS \& C. WACHAMS. 2011. « The Limits of US Assistance to Pakistan » [CAP US Pakistan juillet 2011]. Washington DC : Center for American Progress.

EIZENGS, J. 2012. «A House America Bind for State Housing Finance Agencies » [CAP State Housing mars 2012]. Washington DC : Center for American Progress.

HART, M. 2011. « China Pours Money into Smart Grid Technology » [CAP China Development octobre 2011]. Washington DC : Center for American Progress.

HERSH, A. \& C. WELLER. 2011. « Measuring Future US Competitiveness: US Productivity and Innovation Snapshot » [CAP US Competitiveness février 2011]. Washington DC : Center for American Progress.

JENKINS, J. 2012. «Courting the 'None' Vote: The Religiously Unaffiliated and the Future of American Politics » [CAP Religion novembre 2012]. Washington DC : Center for American Progress.

KENWORTHY, T. \& D. WEISS. 2010. « Drilling Down on Fracking Concerns » [CAP Fracking mars 2010]. Washington DC : Center for American Progress.

KERBY, S. 2012. The State of Women of Color in the United States » [CAP Women of Color juillet 2012]. Washington DC : Center for American Progress.

KORB, L. \& A. ROTHMAN. 2011. « Nation Building at Home » [CAP Nation Building septembre 2011]. Washington DC : Center for American Progress.

KREHELY, J. \& J. HUNt. 2011. « Helping All of Our Homeless: Developing a Gay- and Transgender-Inclusive Federal Plan to End Homelessness » [CAP LGBT Homelessness janvier 2011]. Washington DC : Center for American Progress.

MILHISER, I. 2011. « What If the Tea Party Wins? They Have a Plan for the Constitution and it Isn't Pretty » [CAP Tea Party septembre 2011]. Washington DC : Center for American Progress.

MORGAN, J. 2012. « Making College More Affordable » [CAP College février 2012]. Washington DC : Center for American Progress.

OSHIMA LEE, E., L. ROSENTHAL \& G. SCHEFFER. 2013. « The Effect of Childhood Vaccine Exemptions on Disease Outbreaks » [CAP Health novembre 2013]. Washington DC : Center for American Progress.

SAGAWA, S. 2013. « Service as a Strategy: a Guide for Public Agencies » [CAP Public Agencies février 2013]. Washington DC : Center for American Progress.

SPIRO, T. 2012. « Latest House Republican Budget Threatens Medicare and Shreds the Safety Net » [CAP Safety Net mars 2012]. Washington DC : Center for American Progress.

StACHELBerg, W., A. GERney, C. PARSONS \& M. KNAUSS. 2013. « Preventing Domestic Abusers and Stalkers from Accessing Guns » [CAP Gun Law mai 2013]. Washington DC : Center for American Progress. 
STEIGLEDER, S. \& L. SOARES. 2011. « Harming Our Workforce, Harming Our Nation: Conservatives in Congress Fail to Invest in America's Workforce » [CAP US Workforce avril 2011]. Washington DC : Center for American Progress.

STEINAND, H. \& J. CRAIG. 2013. «Why Austerity Fails When the Cuts Get Specific » [CAP Austerity septembre 2013]. Washington DC : Center for American Progress.

WEISS, D. 2013. « President Obama's Clean Energy Progress » [CAP Energy janvier 2013]. Washington DC : Center for American Progress.

WELT, C. 2010. « Russia, Trade and Human Rights: Thinking through US Policies » [CAP Russia avril 2010]. Washington DC : Center for American Progress.

\subsection{Heritage Foundation}

BAKST, D. \& R. BOCCIA. 2013. « Congress Could Cut Five USDA Programs and Save \$2.8 Billion a Year » [HF Spending juillet 2013]. Washington DC : Heritage Foundation. BLASE, B. 2011. « Medicaid Provides Poor Quality Care: What the Research Shows » [HF Medicaid mai 2011]. Washington DC : Heritage Foundation.

BUCCI, S. 2012. «Getting Cyber Serious: Mastering the Challenges of Federal Cloud Computing » [HF Cyber Security juin 2012]. Washington DC : Heritage Foundation. BURKE, L. \& J. MACNEIL BAKER. 2011. « "Educate to Innovate": How the Obama Plan for STEM Education Falls Short » [HF Education janvier 2011]. Washington DC : Heritage Foundation.

BURKE, L. \& R. SHEFFIELD. 2012. « Obama's 2013 Education Budget and Blueprint; A Costly Expansion of Federal Control » [HF Education avril 2012]. Washington DC : Heritage Foundation.

CARAFANO, J. \& J. RODEBACK. 2010. « Taking the Fight to the Pirates: Applying Counterterrorist Methods to the Threat of Piracy » [HF Piracy mars 2010]. Washington DC : Heritage Foundation.

CHENG, D. \& B. KLINGNER. 2011. « Defense Budget Cuts Will Devastate America's Commitment to the Asia-Pacific » [HF Defense Cuts décembre 2011]. Washington DC : Heritage Foundation.

CURTIS, L. 2012. « Going the Extra Mile for a Strategic US-India Relationship » [HF India août 2012]. Washington DC : Heritage Foundation.

FOSTER, J.-D. 2011. « True Tax Reform: Improves the Economy, Does Not Raise Taxes » [HF Tax Reform novembre 2011]. Washington DC : Heritage Foundation.

GATTUSO, J. 2013. « Taxing Online Sales: Should the Taxman's Grasp Exceed His Reach? » [HF Tax juin 2013]. Washington DC : Heritage Foundation.

GROVES, S. 2013. « US National Human Rights Institution: a Bad Idea » [HF Human Rights novembre 2013]. Washington DC : Heritage Foundation.

HEINRICHS, R. \& B. SPRING. 2012. « Deterrence and Nuclear Targeting in the 21st Century » [HF Nuclear décembre 2012]. Washington DC : Heritage Foundation.

JOHN, D. 2010. « Free the Housing Finance Market from Fannie Mae and Freddie Mac » [HF Housing juillet 2010]. Washington DC : Heritage Foundation. 
KLOSTER, A., L. BURKE \& B. CORONA. 2013. « Department of Justice Uses Decades-old Court Order to Squash Educational Opportunity in Lousiana » [HF Justice septembre 2013]. Washington DC : Heritage Foundation.

LORIS, N. 2013. « US Natural Gas Exports: Lift Restrictions and Empower the State » [HF Gas exports février 2013]. Washington DC : Heritage Foundation.

MICHEL, N. \& J. LIGON. 2010. « Fannie and Freddie: What Record of Success » [HF Finance novembre 2010]. Washington DC : Heritage Foundation.

MORGAN, D. 2013. « Boxer-Sanders Carbon Tax Would Empower EPA to Crush Booming Energy Economy » [HF Energy avril 2013]. Washington DC : Heritage Foundation.

RICHWINE, J. \& J. MARSHALL. 2012. «The Regnerus Study: Social Science on New Family Structures Met with Intolerance » [HF Family octobre 2012]. Washington DC : Heritage Foundation.

Sherk, J. 2011. « How Congress Can Support, Not Hinder, Labor Market Recovery» [HF Labor septembre 2011]. Washington DC : Heritage Foundation.

STIMSON, C. \& S. BUCCI. 2013. « Changing the Military Justice System: Proceed With Caution » [HF Military Mai 2013]. Washington DC : Heritage Foundation.

TYRRELL, P. \& W. BEACH. 2013. « US Government Increases National Debt-and Keeps 128 Million People on Government Programs » [HF Debt janvier 2013]. Washington DC : Heritage Foundation.

UTT, R. 2012. " "Turn Back" Transportation to the States » [HF Transportation janvier 2012]. Washington DC : Heritage Foundation.

ZUCKERMAN, J. 2012. « REAL ID Compliance: Enhancing Security, Respecting Liberty, and Reducing Fraud » [HF Homeland Security novembre 2012]. Washington DC : Heritage Foundation.

\section{Annexe 3. Rapports annuels utilisés pour choisir les trois think tanks étudiés}

« About Brookings ». 2014. Brookings Institution, consulté le 26 mai $<$ www.brookings.edu/about\#research-programs/>.

Center for American Progress. 2011. Doing What Works: Annual Report 2011. Washington DC : Center for American Progress.

\section{NOTES}

1. Le nombre des think tanks américains s'élevait à 1815 en 2012 pour environ 6500 think tanks dans le monde (McGann 2012).

2. Nous notons notamment le rôle des mouvements sociaux des années 1960 ainsi que celui du mouvement conservateur qui cherchait de nouveaux fondements idéologiques pour la pensée conservatrice.

3. Ceci peut également expliquer la forte proportion de think tanks américains dans le monde.

4. Certains, tels que le CEI's Field Guide for Effective Communication consistent essentiellement en une série d'indications pour une diffusion efficace des idées, tandis que d'autres, en l'occurrence l'ouvrage de Raymond Struyk intitulé Managing Think Tanks: Practical Guidance for Maturing 
Organizations, visent à donner des conseils pratiques pour bien organiser un think tank ou encore pour renforcer sa crédibilité et sa notoriété.

5. Certains auteurs tels que Donald Abelson (2006) ou Thomas Medvetz (2012) ont comparé les think tanks américains à des entreprises s'adaptant aux besoins du marché des idées. D. Abelson décrit d'ailleurs ce phénomène comme du « marketing of ideas » (2006).

6. Nous estimons que le personnel de chacun de ces think tanks a augmenté depuis 2005 ; c'est notamment le cas du Center for American Progress qui a travaillé en étroite collaboration avec le président Obama lors de son premier mandat. Ce think tank compte ainsi à présent 95 residentfellows (Center for American Progress 2014).

7. Par «RF », nous entendons « resident fellows » c'est-à-dire les analystes travaillant de façon permanente dans l'organisation. «NRF» désigne les «non-resident fellows »: les analystes travaillant de façon temporaire dans l'organisation. « $\mathrm{E}$ » désigne les « équipiers » c'est-à-dire tout le personnel ne faisant pas de recherche, à savoir le personnel administratif ou le personnel en charge de la communication extérieure.

8. L'adjectif « libérale » désigne l'aile gauche de la politique américaine.

9. Notre corpus compte 223526 mots.

10. Les soulignements des marqueurs d'attitude dans tous les exemples ont été effectués par l'auteur.

11. Nous avons inclus les antonymes de certains mots uniquement quand ils apparaissaient dans notre corpus et que leur contexte d'apparition permettait leur ajout dans un réseau notionnel. Nous n'avons par exemple pas trouvé d'antonymes pour les mots relevant de l'urgence.

12. Sur les 457 marques de première personne de singulier et de pluriel relevées dans notre corpus, seules 55 relèvent de la première personne du singulier. Sur ces 55 occurrences, 29 apparaissent dans des citations et 26 réfèrent aux auteurs.

13. $87 \%$ des documents étudiés obéissent à cette même structure.

14. Le détail non exhaustif des marqueurs d'attitude relevés est présenté en annexe 1.

15. Notons que les termes choisis sont délibérément généraux afin de pouvoir inclure synonymes et antonymes. Le réseau notionnel de l'efficacité implique ainsi des degrés d'efficacité, de même que ceux de la réussite ou de la pertinence.

16. Le contexte entourant le pronom de première personne ne nous a pas toujours permis d'identifier son référent de façon extrêmement précise, d'où la catégorie «Ambigu ». Par "Autre(s)", nous entendons toutes les occurrences de "we» observées dans des citations. En raison de leur forte dispersion au sein de notre corpus, nous n'avons pas pu dégager de réelles tendances pour ce type de marqueur.

\section{RÉSUMÉS}

Cette étude vise à caractériser l'incidence de la position spécifique des think tanks américains à l'interface du monde de la recherche et du monde politique sur leur discours, à travers une analyse structurelle et rhétorique d'un corpus de policy briefs produits par trois think tanks américains entre 2010 et 2013. Elle s'appuie sur l'hypothèse suivante : les contraintes auxquelles sont soumis les experts des think tanks dans leur pratique professionnelle du fait de cette position d'interface et la tension entre expertise et action politique qui en résulte, se reflètent sur certaines caractéristiques du genre. L'analyse structurelle met en lumière la nature codifiée du 
genre organisé en réponse aux besoins d'expertise des hommes politiques. L'analyse rhétorique révèle cependant la dimension programmatique des policy briefs, reflet du besoin de visibilité et d'influence des think tanks dans le monde politique américain. Cette tension entre expertise et visée programmatique, et la nature hybride du genre, permettent de questionner la posture de l'expert des think tanks dans la société américaine.

The present study aims to characterize the impact of American think tanks' specific position - at the crossroads between the academic and the political worlds - on their discourse. It offers a structural and rhetorical analysis of a corpus of policy briefs published by three think tanks between 2010 and 2013. The following hypothesis is explored: the constraints to which think tanks' experts are submitted in their everyday practices due to their position, and the resulting tension between expertise and political action, are reflected in the genre. Results show that the structure of policy briefs is extremely codified since it is organized in response to policymakers' demands for external expertise. However, an analysis of the genre's rhetorical phenomena highlights its programmatic aim, as think tanks offer expertise but also seek to convey their agenda to the political world. Policy briefs thus seem characterized by a hybrid dimension which sheds light on the nature of the expertise offered by American think tanks.

\section{INDEX}

Mots-clés : États-Unis, expertise, genre, policy brief, think tank, visée programmatique Keywords : expertise, genre, policy brief, political agenda, think tank, USA

\section{AUTEUR}

\section{MATHILDE GAILLARD}

Mathilde Gaillard est agrégée d'anglais, élève normalienne en quatrième année au Département des Langues de l'École normale supérieure de Cachan. Sa recherche porte sur le discours des think tanks américains sous l'angle d'une analyse des genres discursifs qu'ils produisent. mathilde.gaillard@ens-cachan.fr 